\title{
Minute Strain Test for Heap Load Scale-down Beam Based on Weak FBG
}

\author{
Yiqiang Yao ${ }^{1}$, Sheng $\mathrm{Li}^{2, *}$ \\ ${ }^{1}$ Key Laboratory of Fiber Optic Sensing Technology and Information Processing, Ministry of Education, \\ Wuhan University of Technology, Wuhan 430070, China; \\ ${ }^{2}$ National Engineering Laboratory for Fiber Optic Sensing Technology, Wuhan University of \\ Technology, Wuhan 430070, China \\ *corresponding author: lisheng@whut.edu.cn
}

\begin{abstract}
Keywords: Scaled-down beam; structural strain; weak FBG; optical fiber ribbon; model experiment.
\end{abstract}
\begin{abstract}
In order to increase the multiplexing capacity of traditional fiber Bragg grating (FBG) sensor and to provide more abundant information support for the structural safety evaluation, structural strain experiment was carried out based on on-line continuous preparation technique for identical and weak FBG. According to uniform strength cantilever beam experiment, good force sensitive linearity and repeatability of naked weak FBG was verified, which indicated the feasibility for structural strain test. The experimental results of scaled-down model showed that the layout configuration for weak FBG was reliable and responses of weak FBG packaged by optical fiber ribbon and theoretical values were consistent in the whole process of minute heap load by masses.
\end{abstract}

\section{Introduction}

Structural strain is an important index for evaluation of load-bearing capacity of civil engineering structure, and according to its results, the strength and stability of structures or components can be acquired ${ }^{[1]}$. Thanks to some positive advantages ${ }^{[2]}$, FBG sensors have become the first choice for long-term strain monitoring and have been widely applied in so many engineering fields ${ }^{[3]-[5]}$.

However, the existing FBG-based sensors have to be processed one by one and then weld together before use, fiber splicing is an inevitable procedure for the application of these FBG, which degrades fiber strength and may cause potential damage to the engineering application.

In order to overcome the above proposed problems which exist in sensor multiplexing and network monitoring in actual engineering, an on-line continuous preparation technique for identical and weak FBG had been reported in references ${ }^{[6][7]}$, which indicated that thousands of weak FBGs could be directly inscribed into fiber during drawing and simultaneously interrogated by proposed methods ${ }^{[8][9]}$. In order to verify the performance of weak FBG under minute load, some pilot experiments about the force sensitive property and wavelength drift deviations compared with theoretical values have been conducted and will be elaborated as follow.

\section{Force sensitive property of weak FBG}

As shown in Fig. 1, a uniform strength cantilever ( $\left.t=3.5 \mathrm{~mm}, L=250 \mathrm{~mm}, D=45 \mathrm{~mm}, E=210 \times 10^{3} \mathrm{MP}_{\mathrm{a}}\right)$ was used to carried out the force sensitive test. In the uniform strength region, traditional FBGs and weak FBGs were stuck on the upper and under surface of the cantilever by epoxy glue. Hook weights composed by four levels were used in loading and unloading processes. The corresponding theoretical strain values were $0, \pm 61 \mu \varepsilon, \pm 175 \mu \varepsilon, \pm 290 \mu \varepsilon$ and $\pm 405 \mu \varepsilon$. Take the measuring points on the upper surface for an example. The responses and differences of wavelength drifts between traditional and weak FBG were shown in Fig.2 depicted by means of three repeated tests. As well as the traditional FBG, weak FBG had good linearity and repeatability. The statistical results showed that force sensitive coefficient ranges for tensile and compressive zones were $1.187 \sim 1.214 \mathrm{pm} / \mu \varepsilon$ and $1.146 \sim 1.231 \mathrm{pm} / \mu \varepsilon$ respectively, which were greater than the result of traditional FBG. The features in compressive zone were almost the same and not to be explained here. 


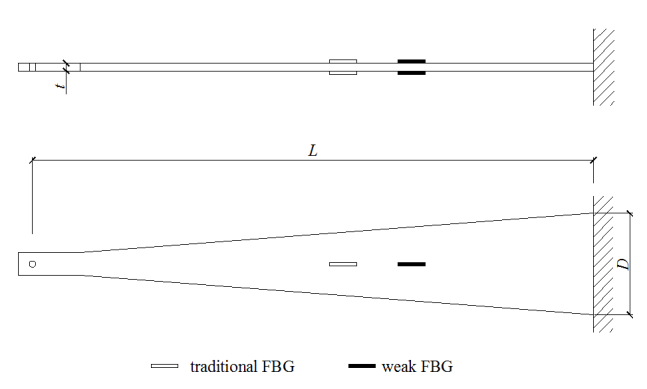

Fig.1 Sketch of uniform strength cantilever beam and FBGs layout

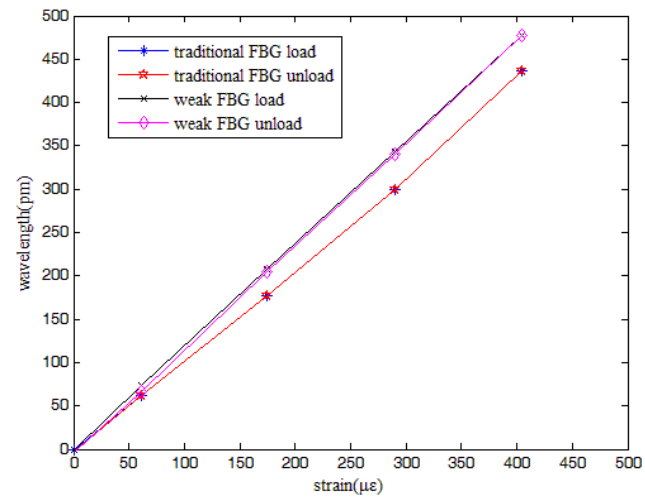

Fig.2 Traditional FBG and weak FBG v.s. theoretical strain

\section{Load experiment for a scale-down beam}

\subsection{Model configuration and sensor layout}

The most common materials in bridge engineering, such as HRB400 steel bar and C50 concrete, were used to cast the beam model. According to similarity principle, $3 \Phi 16$ steel bars were used as longitudinal reinforcements at the location of half-span and bearings. The span length, section size and sensor layout were shown in Fig.3, which totally contained three sections with 18 strain measuring points. By assembling four single optical fiber that contain weak FBG together, the optical fiber ribbon ${ }^{[10]}$ was selected as the packaged means, which effectively increased the spatial resolution and enhanced the mechanical strength of weak FBG. The layout route of one optical fiber ribbon with 18 weak FBGs was similar to Z-shaped and the sensing signal was transmitted by the same optical fiber ribbon. The TDM/WDM technique ${ }^{[11]}$ was adopted to deal with the weak FBG signal.

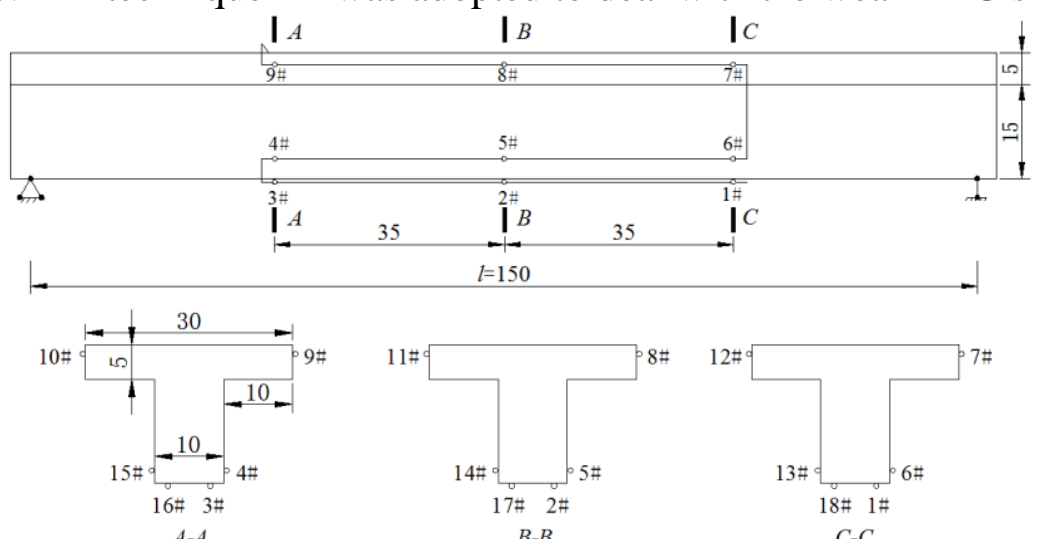

Fig.3 Sketch of scaled-down beam model and strain measuring points layout (unit: $\mathrm{cm}$ )

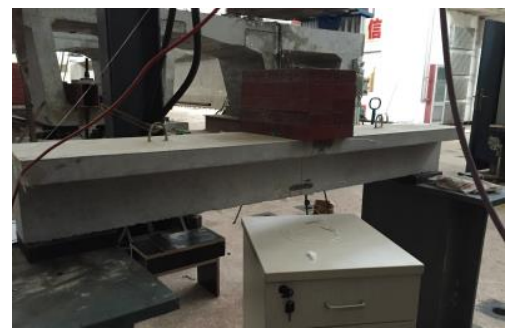

Fig.4 Heap load by 16 masses

\subsection{Loading process}

As shown in Fig.4, load was acted on the surface of top plate at the mid-span section by means of heap load, namely, 16 masses. The weight of each mass was shown in table 1. To test the sensor response, before the formal experiment, preloading was conducted. The number of mass was used to represent the loading level and two masses at each time were defined as the loading or unloading interval. During the whole process, each sub case lasted 5 15 minutes to glean the test data. 
Table 1. Weight of each mass (unit: $\mathrm{kg}$ )

\begin{tabular}{|c|c|c|c|c|c|c|c|}
\hline No. & weight & No. & weight & No. & weight & No. & weight \\
\hline 1 & 10.99 & 2 & 11.00 & 3 & 10.95 & 4 & 10.57 \\
\hline 5 & 11.26 & 6 & 10.80 & 7 & 11.26 & 8 & 11.23 \\
\hline 9 & 10.89 & 10 & 10.89 & 11 & 10.56 & 12 & 10.79 \\
\hline 13 & 10.81 & 14 & 10.55 & 15 & 11.14 & 16 & 10.65 \\
\hline
\end{tabular}

\subsection{Test results}

During the whole loading process, all weak FBG data transmitted by one optical fiber ribbon were recorded completely. Due to the stable temperature field and short loading period, temperature compensation for weak FBG was not considered. According to the previous statistical test, $1.2 \mathrm{pm} / \mu \varepsilon$ was set as the force sensitive coefficient for weak FBG. Take the right measuring points in section B for examples. The strain time-history curves of measuring points 2\#, 5\# and 8\# were depicted in Fig.5. Due to the less precise of heap load and high signal sensitivity of weak FBG, the continuous monitoring curves showed lots of slight oscillations, whereas both the change tendency and the size relationship for the three measuring points were reasonable which conformed to the following equations.

$$
\begin{gathered}
M=P l / 4 \\
\varepsilon=\frac{M y}{E_{c} I_{e q}}
\end{gathered}
$$

Where $M=$ moment of section; $P=$ mass weight; $l=$ span of scale-down beam; $y=$ distance between measuring point and neutral axis; $\varepsilon=$ longitudinal strain; $E_{c}=$ elastic modulus of concrete; $I_{e q}=$ equivalent stiffness of section.

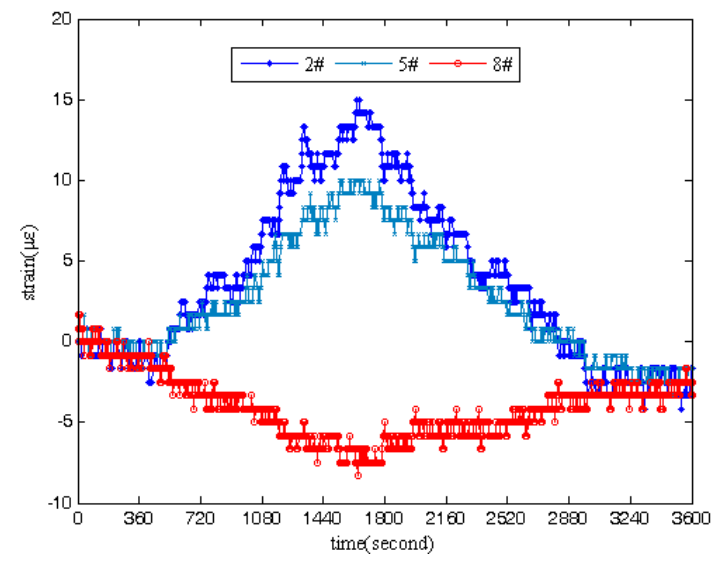

Fig.5 Results comparison of Weak FBG and theoretical value

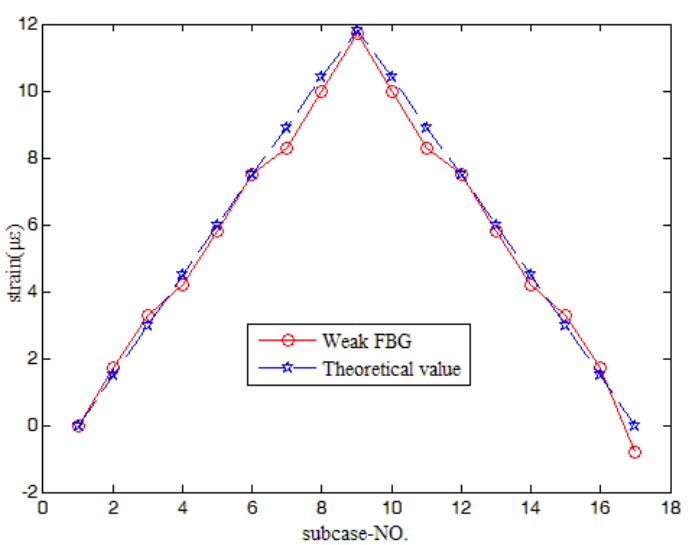

Fig.6 Results comparison of Weak FBG and theoretical value

The theoretical value of $2 \#$ or $17 \#$ was calculated by the above equations and shown in Fig.6. The corresponding average test results of $2 \#$ and 17\# from weak FBG were also given in the same figure. The results showed that the change amplitude and trend for both the test values and the theoretical values were nearly the same and the minute strain can be detected. As well as that, other measuring points showed the same results that the wavelength drift of weak FBG had a good relationship with the loading and unloading process.

\section{Conclusion}

Weak FBG used for structural strain measurement was proposed and the good force sensitive linearity and repeatability of weak FBG and optical fiber ribbon package structure for weak FBG were verified. The results from scaled-down model showed that the proposed layout process for weak FBG was feasible. In addition, the collecting data of weak FBG were integral and stable. Moreover, the 
minute strain responses of weak FBG and theoretical values were consistent during the whole loading process.

\section{Acknowledgements}

The research work reported in this paper was supported by the National Engineering Laboratory for Fiber Optic Sensing Technology, Wuhan University of Technology, China. Thanks to the support of the National Natural Science Foundation of China (Major Program: 61290310).

\section{References}

[1] JTG/T J21-2011. Specification for Inspection and Evaluation of Load-bearing Capacity of Highway Bridges [S] Beijing: China Communications Press, 2011.

[2] D.JIANG \& Richard O. Claus writes. Smart material, instrument, structure and application [M]. Wuhan: Wuhan University of technology press, 2000.

[3] Sheng LI, Junjie MA, JUN Hu. Rockfall Hazard Alarm Strategy Based on FBG Smart Passive Net Structure [J]. Photonic Sensors, 2015, 5(1): 19-23.

[4] Jiang Desheng, Zhou Ciming, Yang Minghong, etal. Research on optic fiber sensing engineering technology [C]. 22rd International Conference on Optical Fibre Sensors, October 15- 19, 2012, Beijing, China.

[5] Li Sheng, Fan Dian, Fu Jinghua, etal. Bridge SHM system based on fiber optical sensing technology [C]. 24th International Conference on Optical Fibre Sensors, September 28 - October 2, 2015, Curitiba, Brazil.

[6] Yu Haihu, ZHENG Yu, GUO Huiyong, etal. Research progress in online preparation techniques of fiber Bragg gratings on optical fiber drawing tower [J]. Journal of Functional Materials, 2014, 45(12): 120001-120005.

[7] YU Hua, HE Wei, GUO Huiyong, LI Xiaofu, etal. Study of Fiber Bragg Grating Fabrication Online on Draw Tower via Phase Mask Method and Equipment Manufacturing [J]. Chinese Journal of Lasers, 2013, 40(5): 0505001-1-0505001-5.

[8] KE Yu'e, ZHENG Yu, LIU Yu, GUO Huiyong etal. Simulation and analysis of reflected signals in identical weak fiber Bragg grating arrays [J]. Study on Optical Communications, 2015, 190 (4):39-41.

[9] LUO Zhihui, CAI Desuo, WEN Hongqiao, GUO Huiyong. A location method for Ultra-Weak Fiber Bragg Grating Array [J]. Acta Optica Sinica, 2015, 35(12): 12606006-1-1206006-5.

[10]CHEN Bingyan writes. Design and Manufacture for Optical Fiber and Fiber Optic Cable [M]. Hangzhou: Zhejiang University Press, 2011.

[11]Wang Chonggang, Wei Wei, Zhang Weiyi, etal. Optimal wavelength scheduling for hybrid WDM/TDM passive optical networks [J]. Journal of Optical Communications and Networking, 2011, 3(6):522-532. 